\title{
Synthesis and characterization of nanometric magnetite coated by oleic acid and the surfactant CTAB
}

\section{Surfactant coated nanometric magnetite/maghemite}

\author{
J. Almazán Celis ${ }^{1}$ - O. F. Olea Mejía ${ }^{1}$ - A. Cabral-Prieto ${ }^{2}$ (D) \\ I. García-Sosa ${ }^{2}$ R. Derat-Escudero ${ }^{3}$ - E. M. Baggio Saitovitch ${ }^{4}$. \\ M. Alzamora Camarena ${ }^{4}$
}

CC Springer International Publishing Switzerland 2017

\begin{abstract}
Nanometric magnetite $\left(n m-\mathrm{Fe}_{3} \mathrm{O}_{4}\right)$ particles were prepared by the reverse coprecipitation synthesis method, obtaining particle sizes that ranged from 4 to $8.5 \mathrm{~nm}$. In
\end{abstract}

This article is part of the Topical Collection on Proceedings of the 15th Latin American Conference on the Applications of the Mössbauer Effect (LACAME 2016), 13-18 November 2016, Panama City, Panama Edited by Juan A. Jaén

\section{A. Cabral Prieto \\ agustin.cabral@inin.gob.mx \\ J. Almazán Celis \\ jony_jac_5@hotmail.com \\ O. F. Olea Mejía \\ oleaoscar@yahoo.com \\ I. García-Sosa \\ irma.garcia@inin.gob.mx \\ R. Escudero Derat \\ escu@unam.mx \\ E. M. Baggio Saitovitch esaitovitch@yahoo.com.br}

M. Alzamora Camarena

mariella.alzamora@gmail.com

1 Centro Conjunto de Investigación en Química Sustentable UAEMéx-UNAM, Universidad Autónoma del Estado de México, Km 14.5 Carretera Toluca-Atlacomulco, San Cayetano, C.P. 50200, Toluca, México

2 Instituto Nacional de Investigaciones Nucleares, Carretera México-Toluca s/n, La Marquesa, Ocoyoacac, Edo. de México, C. P. 52750, México

3 Instituto de Investigación de materiales de la UNAM, Ciudad de México, México

4 Centro Brasileiro de Pesquizas Físicas, Río de janeiro, Brasil 
their synthesis, the concentration of iron salts of ferric nitrate, $\mathrm{Fe}\left(\mathrm{NO}_{3}\right)_{3} \cdot 9 \mathrm{H}_{2} \mathrm{O}$, and ferrous sulfate, $\mathrm{FeSO}_{4} \cdot 7 \mathrm{H}_{2} \mathrm{O}$, were varied relative to the chemical reaction volume and by using different surfactants such as oleic acid (OA) and hexadecyltrimethylammonium bromide (CTAB). The $n m-\mathrm{Fe}_{3} \mathrm{O}_{4}$ particles were characterized by transmission electron microscopy (TEM), Mössbauer spectroscopy (MS), magnetic and X-ray diffraction (XRD) measurements. Typical asymmetrical and/or broad lines shapes appeared in all Mössbauer spectra of the as prepared samples suggesting strong magnetic inter-particle interactions, reducing these interactions to some extent by gentle mechanical grinding. For the smallest particles, maghemite instead of magnetite was the main preparation product as low temperature Mössbauer and magnetic measurements indicated. For the intermediate and largest particles a mixture of magnetite and maghemite phases were produced as the saturation magnetization values of $\mathrm{M}_{\mathrm{S}} \sim 60 \mathrm{emu} / \mathrm{g}$ indicated; these values were measured for most samples, independently of the coating surfactant concentration, and according to the ZFC-FC curves the blocking temperatures were $225 \mathrm{~K}$ and $275 \mathrm{~K}$ for the smallest and largest magnetite nanoparticles, respectively. The synthesis method was highly reproducible.

Keywords Nanomaterials - Magnetite - Maghemite - Magnetic interparticle interactions · Particle agglomeration

\section{Introduction}

The last four decades have witnessed the increasing use of nanometric materials (NMs) in different research and application arenas [1-4]. Such scientific and technological impetus has been associated with the new and promising properties of these NMs. As the particle size decreases below the $100 \mathrm{~nm}$ range, the concomitant morphological particle property change is the increase of its surface-to-volume ratio (SVR). Because of this SVR increase, other physical and chemical properties such as magnetic, structural, catalytic, chemical, etc., change abruptly. Magnetic properties of bulk magnetic materials, with particle size $>500 \mathrm{~nm}$, are far significantly different to those having a particle size $<100 \mathrm{~nm}$. nm- $\mathrm{Fe}_{3} \mathrm{O}_{4}$, for instance, produces more heat than micrometric magnetite $\left(\mu-\mathrm{Fe}_{3} \mathrm{O}_{4}\right)$ under an alternate magnetic field due to a larger magnetic susceptibility. In fact, this magnetic heating property of $n m-\mathrm{Fe}_{3} \mathrm{O}_{4}$ is the basis of magnetic fluid hyperthermia for treating cancers [5-7]. In addition to this, the high biocompatibility of magnetite has also played a crucial role for its large use in other biomedical applications, such as in diagnostics and therapy [8].

Nowadays, a wide variety of chemical recipes to produce $n m-\mathrm{Fe}_{3} \mathrm{O}_{4}$ with any desirable particle size is available in the literature. For instance, a relatively simple procedure to prepare $n m-\mathrm{Fe}_{3} \mathrm{O}_{4}$ with particle sizes between 1 to $10 \mathrm{~nm}$ with low particle size distribution (psd) is by $\gamma$-ray irradiation [9]; this method, however, requires a $\gamma$-ray source installation. Chemical syntheses predominate, co-precipitation being the most widely used method to produce these iron oxides with particle sizes between 5 and $45 \mathrm{~nm}$ but with a relatively large $p s d[10,11]$. In this case the particle size can be adjusted by controlling experimental parameters such as: the initial $\mathrm{Fe}[\mathrm{II}] / \mathrm{Fe}[\mathrm{III}]$ ratio, the nature of the base, $\mathrm{pH}$, method of addition and speed of adding components [12]. More elaborate chemical procedures, such as the organic thermal combustion and micro-emulsion methods have the advantage to produce particles with very low $p s d$ having different crystal forms, ranging from 10 to $50 \mathrm{~nm}$ $[13,14]$. 
Another crucial point that has come out while preparing magnetic nanoparticles (MNPs) is the particle agglomeration due to magnetic inter-particle interactions and a concomitant oxidation process. By using specific organic and/or inorganic species as protective coatings, the agglomeration and oxidation of particles may be prevented [15].

In this sense the present paper describes the preparation of $n m-\mathrm{Fe}_{3} \mathrm{O}_{4}$ prepared by the reverse co-precipitation method where mean particle sizes from 4 to $8.5 \mathrm{~nm}$ were produced. The agglomeration and oxidation processes of $n m-\mathrm{Fe}_{3} \mathrm{O}_{4}$ were studied by using minimal amounts of different organic coatings.

\section{Materials and methods}

$\mathrm{FeSO}_{4} \cdot 7 \mathrm{H}_{2} \mathrm{O}$ was purchased from Sigma-Aldrich, and $\mathrm{Fe}\left(\mathrm{NO}_{3}\right)_{3} \cdot 9 \mathrm{H}_{2} \mathrm{O}$ from MERCK, both of them with $99 \%$ purity. The coatings used were oleic acid (OA) from REASOL and hexadecyl-trimethyl-ammonium bromide (CTAB) from Sigma-Aldrich. These chemicals were used as received.

\subsection{Synthesis of $n m-\mathrm{Fe}_{3} \mathrm{O}_{4}$}

The magnetite synthesis was carried out using the reverse co-precipitation method [10], in which the iron salts, $\mathrm{FeSO}_{4} \cdot 7 \mathrm{H}_{2} \mathrm{O}$ and $\mathrm{Fe}\left(\mathrm{NO}_{3}\right)_{3} \cdot 9 \mathrm{H}_{2} \mathrm{O}$ with an iron molar ratio of 1:2, respectively, were mixed in a beaker with $30 \mathrm{ml}$ of distilled water establishing a $\mathrm{pH}$ of 1 at room temperature. In order to control the particle size, four different concentrations $(\mathrm{mMol} / \mathrm{L})$ of the iron salts were used, such as 1.5:3.0, 3.3:6.6, 4.4:8.8, 6.6:13.2, keeping always the $1: 2$ for the $\mathrm{Fe}(\mathrm{II}) / \mathrm{Fe}(\mathrm{III})$ ratio, respectively. Different concentrations of the surfactants, (OA and $\mathrm{CTAB}$ ) were also used to analyze the particle agglomeration and oxidation processes. One $\mathrm{mMol}$ of these surfactants was added, for the irons concentration ratio of 3.3:6.6 only. For comparative purposes, and keeping the 3.3:6.6 ratio three samples were treated, with zero, one and $10 \mathrm{mMol}$ of CTAB. For the remaining iron concentration ratios, 1.5:3, 4.4:8.8 and 6.6:13.26, zero mMol of surfactant was used, see Table 1. Separately, an alkaline solution was prepared by mixing $6 \mathrm{ml}$ of a $1 \mathrm{M} \mathrm{NaOH}$ solution with $54 \mathrm{ml}$ of distilled water reaching a $\mathrm{pH}$ of 14 and heated to $85^{\circ} \mathrm{C}$ under Nitrogen. Reached this temperature condition and dissolved oxygen removed from this basic solution, the corresponding surfactant was added. Then, the Fe salt solution was added drop wise to the alkaline solution under mechanical steering for 1 hour. The final $\mathrm{pH}$ of the mother reaction solution was 11 for all preparations. At the end of the reaction, the precipitated product was separated by using an external magnet, and it was washed five times with distilled water and methanol to remove residues. Finally, the wet black solid was left to dry under atmospheric conditions for about five hrs. The method reproducibility was tested by preparing the same sample from two to six preparations as indicated in Table 1.

\subsection{MNPs characterization}

Transmission Electron Microscopy (TEM) The morphology, mean particle size and particle size distribution of the as prepared $n m-\mathrm{Fe}_{3} \mathrm{O}_{4}$ particles were analyzed by looking at TEM bright field images. For this purpose a JEOL JEM-2100 model high-resolution TEM, operating at $200 \mathrm{kV}$ was used. 
Table 1 Synthesis parameters of $\mathrm{nm}-\mathrm{Fe}_{3} \mathrm{O}_{4}$ to control its particle size using different surfactants and concentrations

\begin{tabular}{llllllll}
\hline Mode size of $\mathrm{Fe}_{3} \mathrm{O}_{4}(\mathrm{~nm})$ & 4 & 5 & 6 & 6 & 6.5 & 7 & 8.5 \\
\hline Surfactant concentration $(\mathrm{mMol})$ & $1 \mathrm{OA}$ & 0 & $1 \mathrm{CTAB}$ & 0 & 0 & $10 \mathrm{CTAB}$ & 0 \\
$\mathrm{FeSO}_{4} 7 \bullet \mathrm{H}_{2} \mathrm{O}(\mathrm{mMol} / \mathrm{L})$ & 3.3 & 1.5 & 3.3 & 4.4 & 3.3 & 3.3 & 6.6 \\
$\mathrm{Fe}\left(\mathrm{NO}_{3}\right) 3 \bullet 9 \mathrm{H}_{2} \mathrm{O}(\mathrm{mMol} / \mathrm{L})$ & 6.6 & 3.0 & 6.6 & 8.8 & 6.6 & 6.6 & 13.2 \\
Sample & $\mathrm{D}^{\mathrm{a}}$ & $\mathrm{L}^{\mathrm{b}} 3^{\mathrm{b}}$ & L6CTAB $^{\mathrm{c}}$ & L2 $^{\mathrm{d}}$ & L3 $^{\mathrm{e}}$ & L9CTAB $^{\mathrm{f}}$ & L11 $^{\mathrm{g}}$ \\
\hline
\end{tabular}

(a) three syntheses; (b) four syntheses; (c) two syntheses; (d) five syntheses; (e) two syntheses; (f) three syntheses; (g) six syntheses

X-ray diffraction (XRD) The crystal structural characterization of the as prepared $\mathrm{nm}$ $\mathrm{Fe}_{3} \mathrm{O}_{4}$ particles and their mean particle size was analyzed by XRD measurements, using the D5000 diffractometer coupled to a $\mathrm{CuK} \alpha \mathrm{X}$-ray source from Siemens

Mössbauer spectroscopy (MS) The iron oxidation state, the super-paramagnetic and/or ferrimagnetic and agglomeration states of the produced $\mathrm{nm}-\mathrm{Fe}_{3} \mathrm{O}_{4}$ particles were analyzed by MS. Where possible, the corresponding isomer shifts of the sextets were associated with the tetrahedral (A) and octahedral (B) iron sites of the inverse-spinel crystal structure as determined from XRD measurements. The Wissel Mössbauer spectrometer, calibrated with an iron foil $25 \mu \mathrm{m}$ thick, was operating in the constant acceleration mode and a ${ }^{57} \mathrm{Co} / \mathrm{Rh}$ source was mounted on the electromagnetic transducer. Thus, the reported isomer shifts refer to that of metallic iron. Low temperature Mössbauer spectra were also recorded using a Montana cryostat. A homemade least-squares fitting program was used to fit the resulting experimental data.

Magnetic measurements To investigate the blocking temperature of the $n m$ - $\mathrm{Fe}_{3} \mathrm{O}_{4}$ particles, the ZFC (0.0 Oe)-FC (100 Oe) curves were obtained with a SQUID Quantum Design magnetometer from 2 to $300 \mathrm{~K}$. The data of these curves were corrected by considering both the cell and the sample diamagnetic contributions. On the other hand, the saturation magnetization $\left(\mathrm{M}_{\mathrm{S}}\right)$ and the coercive field $\left(\mathrm{H}_{\mathrm{c}}\right)$ were measured through the hysteresis loop by using a Quantum Design VersaLab instrument.

\section{Results and discussions}

\subsection{XRD structural analysis}

Figure 1 shows typical XRD patterns of the as prepared samples, for the largest (Fig. 1a) and smallest (Fig. 1.b) magnetite particle sizes. The XRD pattern for each sample varied on the diffracted line widths only suggesting different crystal sizes. The diffraction peaks can be indexed as a cubic inverse spinel structure of $\mathrm{Fe}_{3} \mathrm{O}_{4}$ (JCPDS no.19-0629), as Fig. 1 shows. The XRD pattern of the smallest particles, Fig. 1b, shows the additional broad peak at around $2 \theta=12$, not indexed; its large intensity is indicating the presence of very small crystalline and/or semicrystalline particles of magnetite/maghemite and/or oxidized particles [7] Thus, a single crystal phase may be present only matching with either magnetite or maghemite crystal structures. In this case, it is difficult to distinguish one phase from another by XRD unless a crystal structure Rietveld refinement analysis is carried out; such 


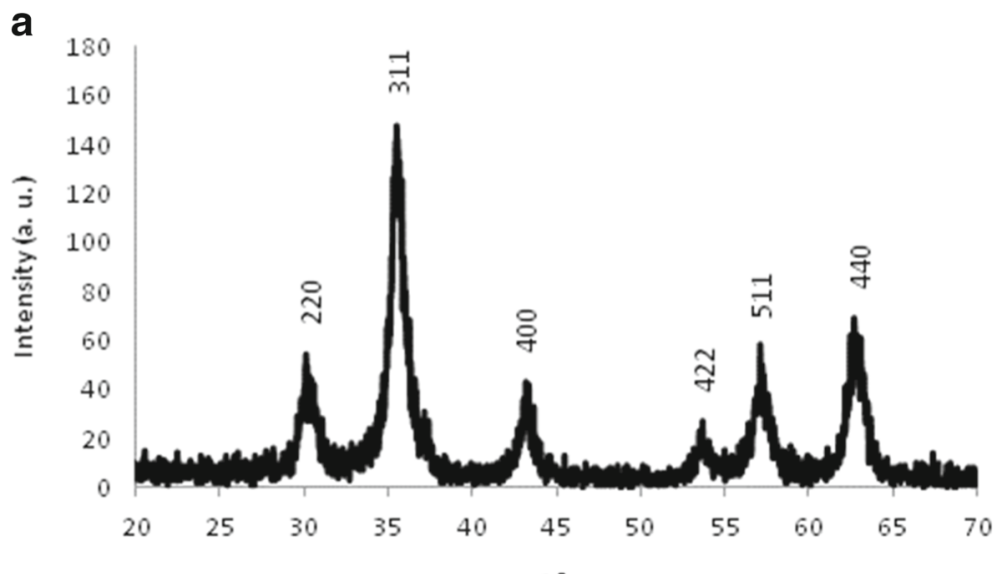

$2 \theta$

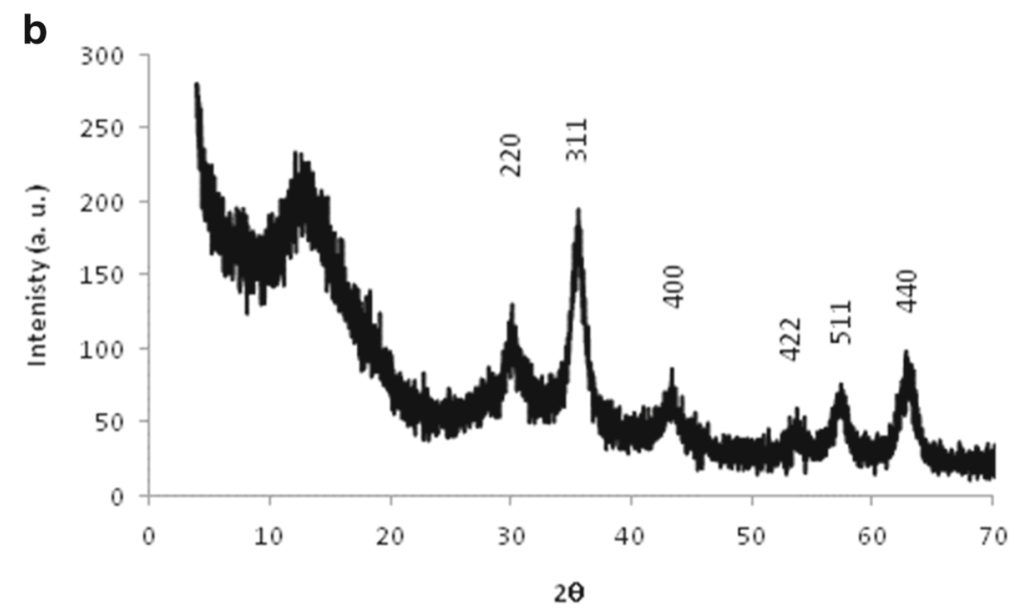

Fig. 1 XRD pattern of nm-Fe ${ }_{3} \mathrm{O}_{4}$ with possible presence of maghemite. The average crystal size for (a) L11 sample was $8.5 \mathrm{~nm}$ as obtained from all indexed peak using the Scherrer's formula. For (b) D5 sample was 4 nm big

an ambiguity was, however, eliminated by using low temperature Mössbauer spectroscopy. The average crystal size, as obtained from the six Miller indexed lines of the XRD patterns, as shown in Fig. 1, and using the Scherrer's equation was for D5 and L11 samples $4 \mathrm{~nm}$ and $8.5 \mathrm{~nm}$, respectively. In order to complement these XRD crystal size determinations, we also used TEM bright field images to assess unambiguously the crystal size and crystal size dispersion of all prepared samples. As known, while XRD data gives information about crystal units, TEM images shows mostly agglomerated particles if not properly dispersed. Data in Table 1 shows the particle size results for the studied samples by TEM, which matched the XRD measurements very closely. As representative examples, Fig. 2 shows the TEM bright field images of three samples showing their mean particle sizes of $8.5 \mathrm{~nm}, 6.5 \mathrm{~nm}, 5 \mathrm{~nm}$ as well as their corresponding frequency histograms (FH's). As seen from the FH's, the samples show certain dispersion with respect to the mean particle size. However, this dispersion is low enough to allow the determination of changes in the magnetic properties as discussed 

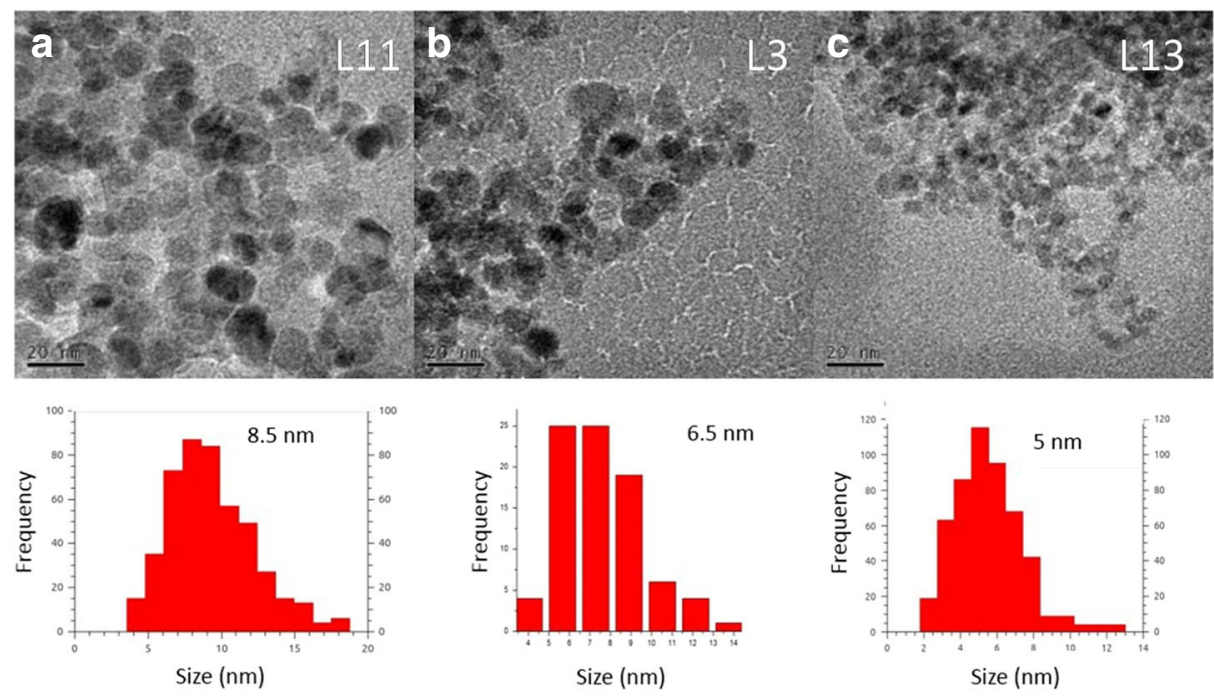

Fig. 2 TEM bright field images as well as their frequency histograms with their mean particle size of (a) L11, (b) L3 and (c) L13 samples

in the next sections. The FH's of 8.5 and $6.5 \mathrm{~nm}$ show slight bimodal distributions, an aspect that will be analyzed in the next section.

\subsection{Mössbauer spectroscopy analysis}

Figure 3a shows the Mössbauer spectrum of the $n m-\mathrm{Fe}_{3} \mathrm{O}_{4}$ particles corresponding to $\mathrm{L} 11$ sample, having the mean particle size of $8.5 \mathrm{~nm}$ according to XRD and TEM measurements, Figs. 1a and 2a, respectively. The first peculiar thing to observe about the Mössbauer spectrum is that at this low particle size of $8.5 \mathrm{~nm}$ one can observe a relatively well resolved but broad magnetic pattern only, suggesting a strong inter-particle interaction [16]. Any sign of superparamagnetism does not appear here. The smeared inward asymmetry of each line appearing in the Mössbauer spectrum of Fig. 3a is, on the other hand, the characteristic spectral feature for inferring magnetic inter-particle interactions $[17,18]$. In the absence of such an interaction, the Mössbauer spectrum must show the typical superparamagnetic behavior for such small nanoparticles (NPs). When using a discrete Mössbauer parameter analysis for this Mössbauer spectrum, the best fit resulted in three magnetic sub-patterns.

The narrower patterns could be associated with typical $\mathrm{Fe}^{3+}$ in tetrahedral $\mathrm{A}$ sites and $\mathrm{Fe}^{2+}$ and $\mathrm{Fe}^{3+}$ in octahedral B sites of the cubic inverse spinel structure of magnetite, with isomer shifts $(\delta)$ of $0.33 \mathrm{~mm} / \mathrm{s}$ and $0.42 \mathrm{~mm} / \mathrm{s}$, respectively. The isomer shift of B sites, $\delta=0.42 \mathrm{~mm} / \mathrm{s}$, is the average isomer shift of the ferrous and ferric cations in B sites, as indicated in the inset data of Fig. 3a. The broader line widths of the sextet associated with the B sites is also typical for magnetite. Such a broadening has been associated with the presence of several fields at the $\mathrm{B}$ site rather than a relaxation process between $\mathrm{Fe}^{2+}$ and $\mathrm{Fe}^{3+}$ as initially thought [19]. On the other hand, the relative spectral areas ratio of these sextets deviates from the stoichiometric ratio of $1(\mathrm{~A}): 2$ (B) to $1(\mathrm{~A}): 1.83(\mathrm{~B})$. Such a deviation must come from several factors. For instance, the Mössbauer fractions for the A and $\mathrm{B}$ sites are probably slightly different, having lower fractions the $\mathrm{Fe}^{2+}$ and $\mathrm{Fe}^{3+}$ ions 


\section{a}


Fig. 3 Mössbauer spectra of magnetite L11 and from a thermal decomposition prepared sample with (a) 8.5 $\mathrm{nm}$ and (b) $23 \mathrm{~nm}$ mean crystal sizes. The units of $\gamma, \delta \mathrm{E}$ and $\Gamma$ are $\mathrm{mm} / \mathrm{s}$

in the B octahedral sites. Vacancies are also typical in nanostructures and probably the B sites, more exposed to the surface particle, would have more crystal defects than the more structurally internal A sites, generating structural changes around the Fe ions in B sites [18]. Both these factors would decrease the spectral area of B sites. Finally, one may argue that these sextets arise from maghemite rather than from pure magnetite because of the close proximity of the isomer shifts of these sextets. Others may argue that the experimental Mössbauer spectrum of Fig. 3a arises from a mixture of both magnetite and maghemite. This possibility may be close to reality even though the co-precipitation reaction took place under the typical alkaline and under oxygen free conditions. Others would also say that the small $\delta$ value for B sites is due to the smallness of the crystallites. Thus, the proximity of the isomer shifts of 0.33 and $0.42 \mathrm{~mm} / \mathrm{s}$ of these narrow sextets probably results from both the small particle sizes and the possible presence of maghemite. For comparative purposes, Fig. $3 \mathrm{~b}$ shows the Mössbauer spectrum of a $\mathrm{nm}-\mathrm{Fe}_{3} \mathrm{O}_{4}$ with a mean particle size of 23 $\mathrm{nm}$ produced by thermal combustion. As seen from the inset data in Fig. $3 \mathrm{~b}$ the relative intensities of the characteristic magnetite sextets is 1.82 , being practically the same as the reported in Fig. 3a with a value of 1.83 . In this case the resulting isomer shifts $(\delta)$, being 
0.33 and $0.28 \mathrm{~mm} / \mathrm{s}$ associated with $\mathrm{Fe}^{3+}$ in A sites, and 0.42 and $0.64 \mathrm{~mm} / \mathrm{s}$ with $\mathrm{Fe}^{2+}$ and $\mathrm{Fe}^{3+}$ in $\mathrm{B}$ sites of Fig. 3a and b, respectively. The marked difference in $\delta$ values for the B sites in these samples may suggest that the sample having the larger particle size is less maghemite contaminated than the sample having smaller particle size. It is worth noting that the line width $(\Gamma=1.04 \mathrm{~mm})$ of the B sextet in Fig. 3a is larger to that of the corresponding B sextet $(\Gamma=0.45 \mathrm{~mm} / \mathrm{s})$ in Fig. 3b. Such a difference clearly suggests a particle size effect and/or a different Mössbauer fraction of maghemite. As shown in Fig. 4, when the magnetic pattern of these magnetites collapses into a broad quadrupole doublet or a broad singlet, the existing difference between isomer shifts associated with the A and B sites vanishes, and a single isomer shift value appears being characteristic of $\mathrm{Fe}^{3+}$ with $\delta \sim 0.33 \mathrm{~mm} / \mathrm{s}$ only. A partial spectral magnetic collapse into a superparamagnetic quadrupole doublet is observed in Fig. 3b, where the isomer shift of this doublet, of $\delta=0.43 \mathrm{~mm} / \mathrm{s}$, is still indicative of the presence of $\mathrm{Fe}^{2+}$ in $\mathrm{B}$ sites. One thus may infer from these considerations that the proximity of the isomer shifts for A and B sites appearing in Fig. 3.a is mainly due to particle size effects rather than to maghemite contamination.

The third broad magnetic sextet appearing in Fig. 3a, on the other hand, which by the way has a $\delta=0.4 \mathrm{~mm} / \mathrm{s}$ also indicative of the presence of $\mathrm{Fe}^{2+}$, may give us a qualitative and simple measure of the agglomeration state of these nanoparticles, an aspect that for example must be minimized for biomedical applications. Assuming similar Mössbauer fractions for all iron sites in L11 sample, Fig. 3a, about $53 \%$ of the particles should show strong magnetic inter-particle interactions. For some authors this broad magnetic sextet would represent amorphous material [20], which does not apply here. The smeared inward asymmetry of each line appearing in the Mössbauer spectrum of Fig. 3a, as already mentioned, is the characteristic spectral feature for inferring magnetic inter-particle interactions in nanomaterials $[18,19]$ and this interaction could qualitatively be measured by the relative intensity of this broad sextet. M. Polikarpov et al. [21] has recently theoretically evaluated the line asymmetry of the Mössbauer lines of $\gamma-\mathrm{Fe}_{2} \mathrm{O}_{3}$ making use of the multi-level relaxation model, from which the energy barrier $\left(\mathrm{E}_{\mathrm{b}}\right)$ is calculated. The larger the $\mathrm{E}_{\mathrm{b}}$ value, the bigger is the cluster of agglomerated nanoparticles and less effective to generate heat. $\mathrm{E}_{\mathrm{b}}$ is connected with the Neel formula for relaxation of the magnetization vector of a superferromagnetic cluster and is proportional to the barrier height that the magnetization vector has to overcome in order to reverse its orientation [21].

We can also observe such a line asymmetry for all other Mössbauer spectra of the prepared magnetite nanoparticles either with small or large particle sizes, as shown in Fig. 4. Generally speaking and assuming a thin absorber approximation, symmetrical Lorentzian or at most Voigtian lines shapes are characteristic of nanoparticles not showing interparticle interaction, which is not the case for any Mössbauer spectrum shown in Fig. 4, thus suggesting strong magnetic inter-particle interaction for all prepared samples.

While trying to understand the agglomeration and/or the magnetic inter-particle interaction among particles of our samples, there is no clear evidence that the iron concentration ratios used in the preparation of these NPs and/or the amount of surfactants used, defined the agglomeration of particles. For instance, the Mössbauer spectrum of D5 sample, having the smallest mean particle size, Fig. 4a, was prepared by using the $\mathrm{Fe}(\mathrm{II}) / \mathrm{Fe}(\mathrm{III})$-ratio concentration of 3.3:6.6 and one mMol of oleic acid, as indicated in Table 1. A practically similar Mössbauer spectrum (not shown) for L13 sample was obtained, which was prepared by using the $\mathrm{Fe}$ (II)/Fe(III)-ratio concentration of 1.5:3. By using the same discrete Mössbauer parameter analysis, broad patterns, a quadrupole doublet and a sextet were used to fit the Mössbauer spectra of samples D5 and L13. The relative intensities of the sextets were $70 \%$ and $67 \%$ for D5 and L13 samples, respectively. These percentages suggest about the same 
Fig. 4 Mössbauer spectra of the $\mathrm{nm}-\mathrm{Fe}_{3} \mathrm{O}_{4}$ with particle sizes from (a) $5 \mathrm{~nm}$ (L13), (b) $6 \mathrm{~nm}$ (L6CTAB), (c) $6 \mathrm{~nm}$ (L2) and (d) 7nm (L9CTAB10) samples
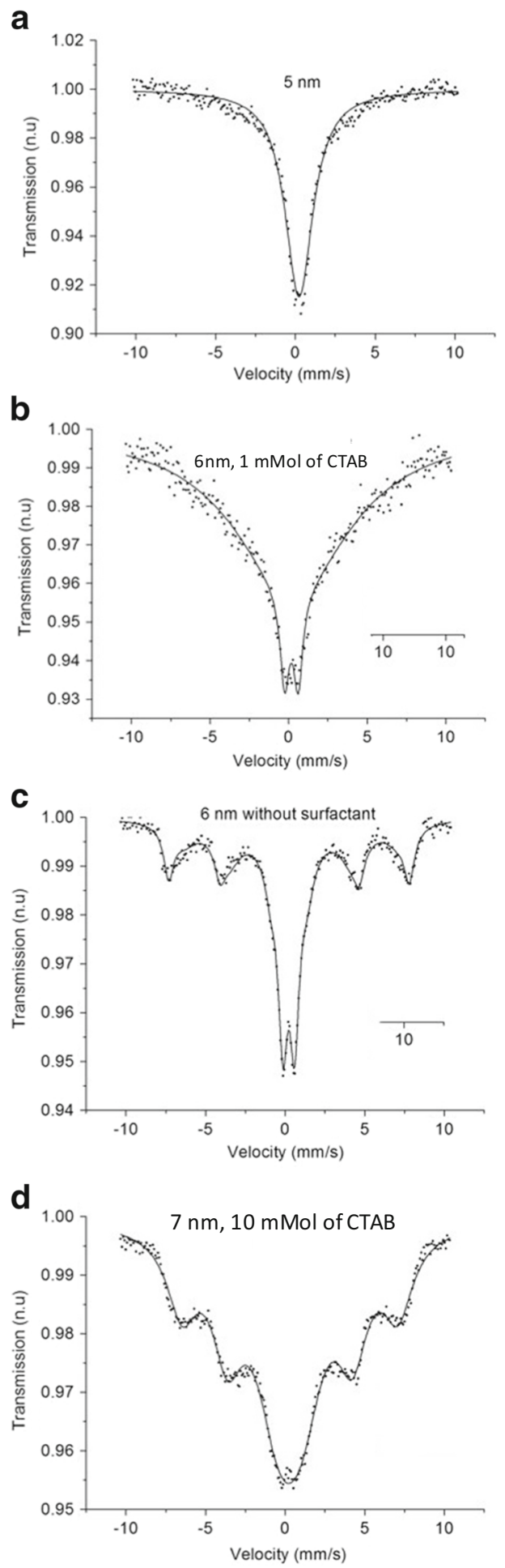
degree of magnetic inter-particle interaction and/or agglomeration, suggesting that under the present experimental conditions neither the irons concentration ratio nor the presence or absence of surfactant affected greatly the agglomeration and/or inter-particle interaction.

The Mössbauer spectrum shown in Fig. $4 \mathrm{~b}$ is due to the L6CTAB sample as prepared with the $\mathrm{Fe}(\mathrm{II}) / \mathrm{Fe}$ (III)-ratio concentration of 3.3:6.6 in presence of $1 \mathrm{mMol}$ of CTAB and having a mean particle size of $6 \mathrm{~nm}$, as indicated in Table 1. At first glance, one observes a Mössbauer spectrum having a line broadening larger than that of Fig. 4a. Similarly, the best fit to this spectrum were two broad patterns, a superparamagentic singlet and a magnetic sextet. Assuming the latter to be the cause of magnetic inter-particle interaction, one gets a $10 \%$ and $90 \%$ contributions for the former and latter patterns, respectively. There appears to be, in this case, an increase of inter-particle interaction because of the presence of the CTAB surfactant only.

Before discussing Fig. 4c, it is of particular interest to discuss the resulting Mössbauer spectrum of the L9CTAB10 or (L15-2) sample, having a mean particle size of $7 \mathrm{~nm}$ and prepared under the presence of $10 \mathrm{mMol}$ of CTAB surfactant, see Figs. $4 \mathrm{~d}$ and/or 5c. These spectra are the result of two different sample preparations using the same chemical conditions. The broad but relatively well resolved magnetic sextet, as compared to the previous ones of Fig. 4a, b and c, should result, at first sight, form an even more agglomerated sample. As observed from the data of Table 1, the slight increase of the measured mean particle size from 6 to $7 \mathrm{~nm}$ for samples L6CTAB and L9CTAB10, respectively, was due to the different amount of surfactant only. One of the best fits to these spectra is shown in Fig. 5c, where two broad magnetic patterns were needed to fit these spectra with hyperfine magnetic fields of $19.4 \mathrm{~T}$ and $40.4 \mathrm{~T}$ and relative intensities of 55 and $45 \%$, respectively, as indicated in the inset data of Fig. 5c.

Two possibilities are considered to understand the origin of these magnetic patterns in L9CTAB10 sample: (1) the presence of the magnetic inter-particle interaction or (2) the presence of a bimodal particle size distribution. The L9CTAB10 sample was gently mechanically grinded using an agate mortar for one minute, see Fig. 5b. The relatively well-resolved sextet for the un-grinded sample became in a less resolved sextet for the grinded sample, being an indication that such a gentle mechanical grinding resulted in a slightly reduced magnetic inter-particle interaction and not due to a bimodal particle size distribution. The possibility of an oxidation process of magnetite because of the mechanical grinding may be discarded; at most, magnetite would transform into maghemite and the resulting Mössbauer spectrum after grinding should be practically unchanged. The transformation of magnetite to maghemite seems also unlikely because this sample was highly coated.

Contrary to this case, now let us go back and look at the Mössbauer spectrum shown in Fig. 4c, arising from L2 sample as prepared by using an $\mathrm{Fe}(\mathrm{II}) / \mathrm{Fe}(\mathrm{III})$-ratio concentration of 4.4:8.8 without surfactant. Figure 2 shows the corresponding FH's for some of the studied samples, suggesting the presence of a slightly bimodal particle size distribution. This bimodal particle-size-distribution feature was typical for practically all prepared samples. Such a feature was, however, less pronounced for the smallest particles as can be seen from Fig. 2. The FH for L2 sample was quite similar to that shown for L3 sample as indicated in Fig. 2. The best fit for the Mössbauer spectrum shown in Fig. 4c or Fig. 5a consisted of two magnetic patterns and a quadrupole doublet. As in Fig. 3, the fitted magnetic patterns are characteristic of magnetite with isomer shifts of 0.34 and 0.47 $\mathrm{mm} / \mathrm{s}$ for the A and B sites already identified, with hyperfine magnetic fields of $47 \mathrm{~T}$ and $41 \mathrm{~T}$ for $\mathrm{A}$ and $\mathrm{B}$ sites, respectively. The hyperfine Mössbauer parameters of the quadrupole doublet were $\delta=0.33 \mathrm{~mm} / \mathrm{s}$ and $\Delta \mathrm{E} / 2=0.78 \mathrm{~mm} / \mathrm{s}$, the rest of the Mössbauer parameters are shown in Fig. 5a. Since this Mössbauer spectrum seems to present the lowest line 

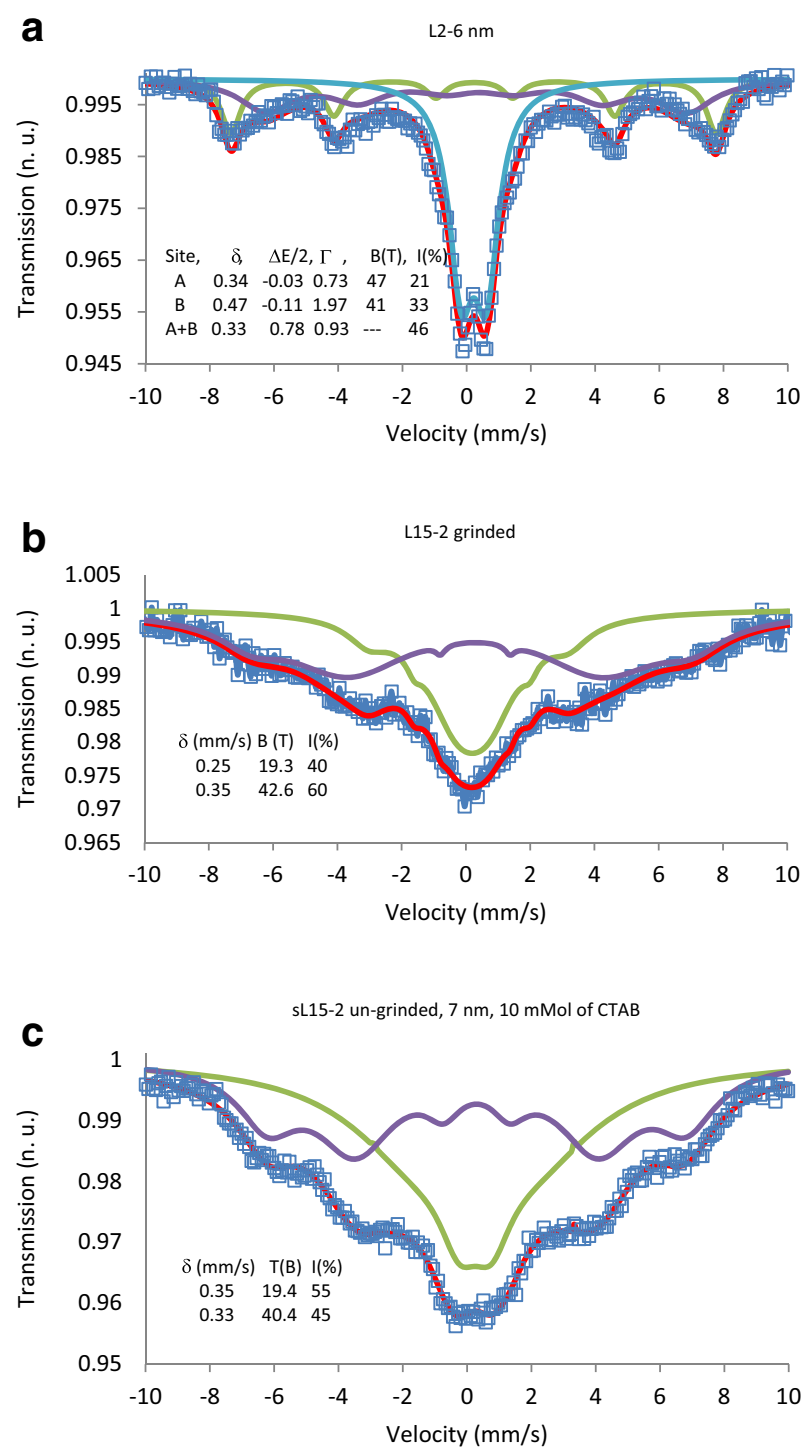

Fig. 5 Mössbauer spectra of (a) L2, (b) L15-2 mechanically grinded and (c) un-grinded samples

shape asymmetry as compared to those in Fig. 4a, b and d, there exists the possibility that this spectrum may arise from a bimodal particle size, as already suggested. Another possibility is the core-shell structure with an oxidized shell. The shell would contribute with part of the quadrupole doublet. The inward asymmetrical lines of the magnetic components in Fig. 4c, suggesting magnetic inter-particle interaction and therefore particle agglomeration was not further investigated, an aspect that will be studied with more detail in the near future. Low temperature Mössbauer measurements would also assess the nature of the quadrupole doublet in Fig. 4c. At present, the origin of this doublet was associated with NPs of magnetite/maghemite only as indicated in the inset data of Fig. 5a. 


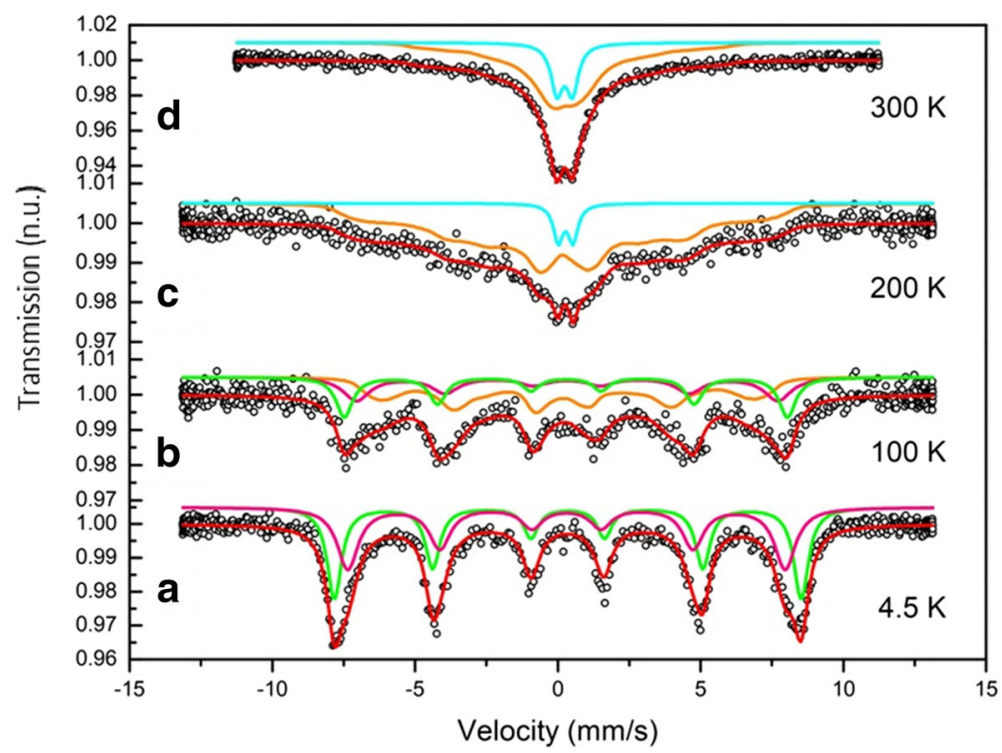

Fig. 6 Mössbauer spectra of L13 sample with mean particle size of $5 \mathrm{~nm}$ at (a) 4.5K, (b) 100K, (c) $200 \mathrm{~K}$ and (d) $300 \mathrm{~K}$

On the other hand, in order to define the nature of the samples having the smaller particles sizes, where there is an indication of oxidation from our previous analysis, Mössbauer spectra of L13 sample were recorded at 4.5K, 100K, 200K and 300K, as shown in Fig. 6. The Mössbauer spectrum recorded at $4.5 \mathrm{~K}$, Fig. 6a, consists of well resolved two sextets only, whose isomer shifts for A sites is $0.39 \mathrm{~mm} / \mathrm{s}$ and for B sites $0.38 \mathrm{~mm} / \mathrm{s}$, being practically the same and therefore suggesting the presence of $\mathrm{Fe}^{3+}$ cations in both $\mathrm{A}$ and $\mathrm{B}$ sites of the cubic spinel crystal structure. This indicates that this L13 sample consists of maghemite only. These low temperature Mössbauer measurements were recorded at least two months after the samples preparation, suggesting that a full oxidation of the samples may have taken place after this relatively short period, or that it is a feature of the reverse co-precipitation method while producing such small particles. As seen from this $4.5 \mathrm{~K}$ Mössbauer spectrum there is no spectral signs of a core-shell structure. If oxidation occurs, the magnetite transforms to maghemite only.

The Mössbauer spectrum recorded at 100K, Fig. 6b, shows, on the other hand, a broad magnetic sextet without any signal of superparamagnetism yet. It can also be observed from the fitting analysis that three magnetic patterns were required to fit the spectrum, two of them associated with the A and B sites as already discussed and the third one, with the lower hyperfine magnetic field, can be associated with the magnetic inter-particle interaction. The Mössbauer spectrum recorded at 200K, Fig. 6c, shows, however, the signs of superparamagnetism and ferrimagnetism. The blocking temperature for this L13 sample is about $225 \mathrm{~K}$ as seen next.

\subsection{Magnetic measurements}

To investigate further the nature of our magnetic samples, some magnetic hysteresis and ZFC-FC curves were recorded. Figure 7 shows the resulting curves for two selected samples only. 

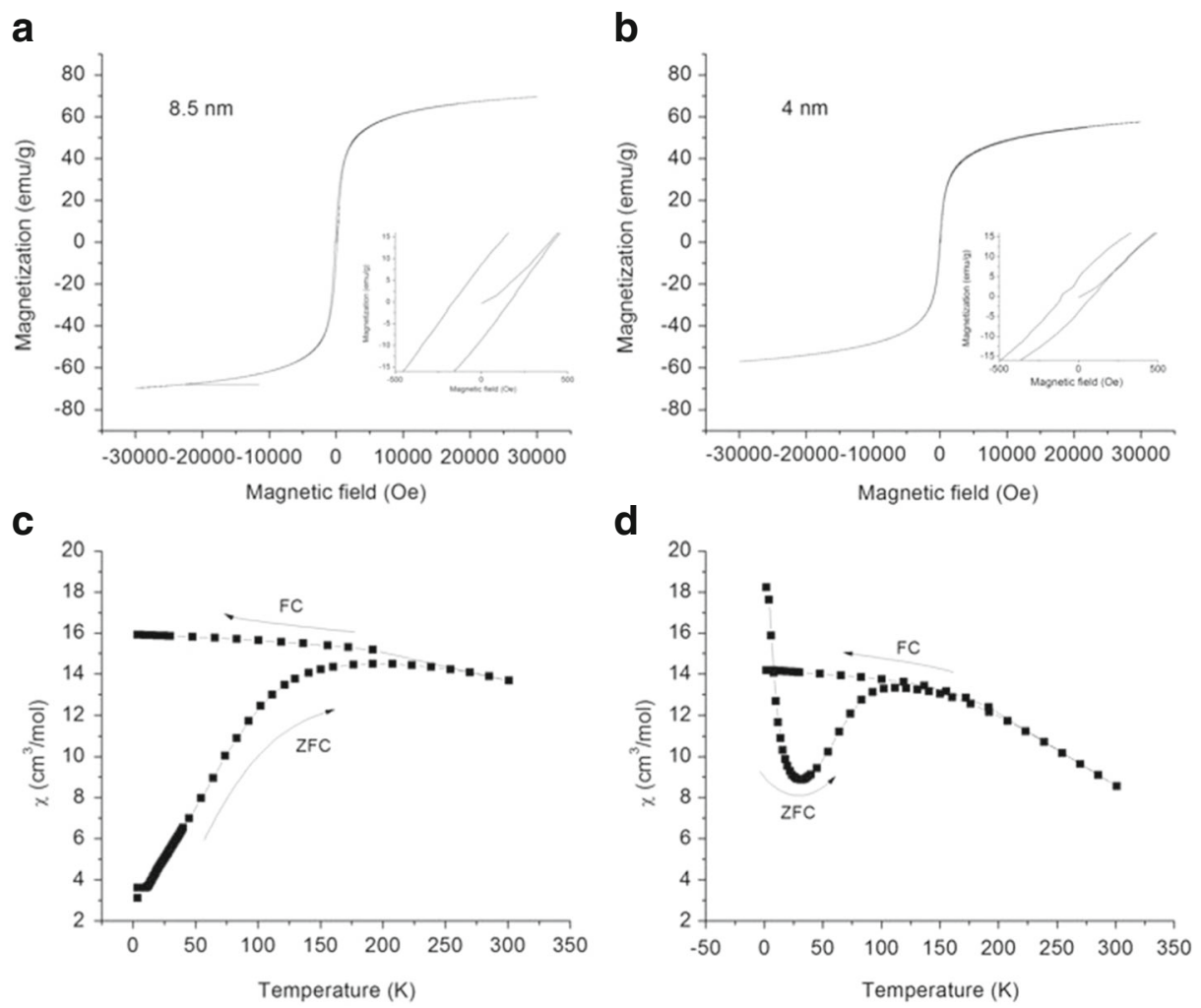

Fig. 7 Hysteresis (a and b) and ZF-ZFC curves (c and d) of samples having the biggest and lowest particle sizes

One thing to observe is that the ZFC curve for one of the samples having the smallest particle sizes, the L13 sample, Fig. 7d, is quite different in the low temperature region from that of the sample having the largest particle size, the L11 sample, Fig. 7c. The low temperature magnetic features of the L13 sample and observed in other nanomaterials arise from spin structures freezing [22]. The most peculiar fact is that this sample has a large magnetic susceptibility $(\chi)$ at the lowest temperature which decreases as the temperature increases (the unfreezing process). A minimum forms and then $\chi$ begins to increase in the normal way as the temperature increase reaching an inflection point at $120 \mathrm{~K}$ for the bigger particles and at $100 \mathrm{~K}$ for smaller particles. That is, before reaching the inflection temperature values the magnetic inter-particle interactions are strong enough to align many particles or magnetic domains themselves. The typical ZFC curve showing the Verwey transition is not observed here suggesting the absence of this transition [11,23]. After reaching the maxima, at $T_{P}$ the $\chi$ values begin again to decrease, where thermal effects begin to predominate over the magnetic inter-particles interactions, and both curves, the ZFC and FC, touch each other defining at this point the blocking temperature $\left(\mathrm{T}_{\mathrm{B}}\right)$. These temperatures are $225 \mathrm{~K}$ and $275 \mathrm{~K}$ for L13 and L11, respectively, at which the relaxation time of the particles magnetic vector is the same as the time measurement. Below $\mathrm{T}_{\mathrm{B}}$ the particles are ferrimagnetic and above $T_{B}$ are superparamagnetic. One can observe from the Mössbauer spectrum recorded at $200 \mathrm{~K}$, Fig. 6c, a mixed pattern, a broad superparamagnetic doublet and a broad ferrimagnetic sextet, with about similar intensities. One should also notice that even though these 
magnetic measurements indicated us that above $225 \mathrm{~K}$ or $275 \mathrm{~K}$ the particles are superparamagnetic, this feature did not occur for most samples; in particular, for those having sizes $>7$ $\mathrm{nm}$. As already discussed, which was mainly due to the strong inter-particle magnetic interactions of the agglomerated particles. On the other hand, the saturation magnetization $\left(\mathrm{M}_{\mathbf{S}}\right)$ of these samples are below the characteristic value for pure magnetite $(80 \mathrm{emu} / \mathrm{g})$, being 60 emu/g and $57 \mathrm{emu} / \mathrm{g}$ for L11 and L13 samples, respectively, Fig. 7, which are characteristic values for oxidized magnetite [24]. It is worth mentioning that the $\mathrm{M}_{\mathrm{S}}$ values of these and the rest of the studied samples did not varied too much from these values suggesting that the surfactants coating in some cases was very little but in other cases enough to change the crystal size. Finally, it can be observed from the inset figures of Fig. 7a and b, narrow hysteresis loops for both samples. Such a feature should not appear for these small particles. One thus must infer that the corresponding small coercive fields $\left(\mathrm{H}_{\mathrm{C}}\right)$, higher for the larger particles, must come form the agglomerated clusters as a whole and not form individual crystals or magnetic mono domains. The $\mathrm{H}_{\mathrm{c}}$ values may possibly give a qualitative measure of the magnetic inter-particle interaction and/or agglomeration.

\section{Conclusions}

The reverse synthetic co-precipitation chemical method, used to produce magnetite/maghemite with particle sized that ranged from 4 to $8.5 \mathrm{~nm}$ in a controlled manner was highly reproducible. The particle sizes as measured by both XRD and TEM bright field images were in good agreement. Under the present experimental reaction conditions, the lowest particle sizes of maghemite were produced by using the lower concentrations of the iron salts with and without any surfactant. On the other hand, the bigger particle sizes were produced by using the larger iron concentrations. In all cases and under our experimental conditions, the presence of the surfactants had the effect of reducing the particle size, the particle size distribution and the particle agglomeration. Except one sample, all other samples presented a strong inter-particle magnetic interaction as inferred from the Mössbauer spectra. This inter-particle interaction was slightly reduced by mechanically grinding. Furthermore, from low temperature Mössbauer spectra and saturation magnetization measurements it was concluded that maghemite rather than magnetite was formed while preparing the smallest particles sizes. For the intermediate and larger particles sizes a mixture of magnetite/maghemite particles were always produced as evidenced from saturation magnetic measurements. Strong magnetic inter-particle interaction occurred in most of the prepared samples and prevented the observation of superparamagnetism, particularly in sample showing particles sizes above $7 \mathrm{~nm}$.

Acknowledgments To CONACyT by the master scholarship. To ININ for allowing me to work on their premises. To CBPF for the researches.

\section{References}

1. Wei, W., He, Q., Jiang, C.: Magnetic iron oxide nanoparticles: synthesis and surface functionalization strategies. Nanoscale Res. Lett. 3, 397-415 (2008)

2. Dave1, P.N., Chopda, L.V.: Journal of Nanothecnology. doi:10.1155/2014/398569 (2014)

3. Xingu-Contreras, E., García-Rosales, G., García-Sosa, I., Cabral-Prieto, A., Solache-Ríos, M.: Characterization of natural zeolite clinoptilolite for sorption of contaminants. Hyperfine Interact. 232(1), 7-18 (2015) 
4. Hilger, I., Kaiser, W.A.: Iron oxide-based nanostructures for MRI and magnetic hyperthermia. Nanomedicine 7(9), 1443-1459 (2012)

5. Rosensweig, R.E.: Heating magnetic fluid with alternating magnetic field. J. Magn. Magn. Mater. 252, 370-374 (2002)

6. Adhikary, K., Banerjee, M.: Journal of Nanoparticles. doi:10.1155/2016/6309231 (2016)

7. Donglu Shi, M.E., Sadat, A.W., Mast, D.D.B.: Nanoscale. doi:10.1039/c5nr01538c (2015)

8. Lewinski, N., Colvin, V., Drezek, R.: Cytotoxicity Nanopart., small 4(1), 26-49 (2008)

9. Abedini, A., Razak Daud, A., Hamid, M.A.A., Othman, N.K.: Cytotoxicity of nanoparticles. PLOS one 9(3) (2014). doi:10.1371/journal.pone.0090055

10. Mamani, J.B., Costa-Filhob, A.J., Cornejoc, D.R., Vieirad, E.D., Gamarraa, L.F.: Mater. Charact. 81, 28-36 (2013)

11. Baumgartner, J., Bertinetti, L., Widdrat, M., Hirt, A.M., Faivre, D.: Synthesis and characterization of magnetite nanoparticles coated with lauric acid. PLOS one. doi:10.1371/journal.pone.0057070 (2013)

12. Davies, K.J., Wells, S., Charles, S.W.: J. Magn. Magn. Mater. 122, 24-28 (1993)

13. Zhichuan, X., Shen, C., Tian, Y., Shi, X., Gao, H.-J.: The effect of temperature and oleate adsorption on the growth of maghemite particles. Nanoscale 2, 1027-1032 (2010)

14. Zhang, J., Qiu, S., Zhu, Y., Huang, Z., Yang, B., Yang, W., Mingyuan, W., Qingyun, W., Yang, J.: Organic phase synthesis of monodisperse iron oxide nanocrystals using iron chloride as precursor. Polym. Chem. 4, 1459-1466 (2013)

15. Ningthoujam, R.S., Vatsa, R.K., Kumar, A., Pandey, B.N.: Barc Newsl. 323, 18-23 (2011)

16. Thanh, H.N., Dai, L.T., Hung, M.D., Vinh, H.T., Van, H.L., Xuan, P.N.: Adv. Nat. Sci.: Nanosci. Nanotechnol. 1(7pp), 035001 (2010)

17. Polikarpov, M., Cherepanov, V., Chuev, M., Shishkov, S., Yakimov, S.: ICAME 2009 J. Phys. Conf. Ser. 217. doi:10.1088/1742-6596/217/1/012114 (2010)

18. Mørup, S., Fougt Hansen, M., Frandsen, C.: Beilstein J. Nanotechnol. 1, 182-190 (2010)

19. Brok, E., Frandsen, C., Madsen, D.E., Jacobsen, H., Birk, J.O., Lefmann, K., Bendix, J., Pedersen, K.S., Boothroyd, C.B., Berhe, A.A., Simeoni, G.G., Mørup, S.: J. Phys. D: Appl. Phys. 47, 365003 (2014)

20. Drbohlavova, J., Hrdy, R., Adam, V., Kizek, R., Schneeweiss, O., Hubalek, J.: Sensors 9, 2352-2362 (2009)

21. Polikarpov, M., Cherepanov, V., Chuev, M., Gabbasov, R., Mischenko, I., Jain, N., Jones, S., Hawkett, B., Panchenko, V.: J. Magn. Magn. Mater. 380, 347-352 (2015)

22. Long, G.J. (ed.): Mössbauer Spectroscopy Applied to Inorganic Chemistry, vol. 1. Plenum Press, New York (1984)

23. Lee, J., Kwon, S.G., Park, J.-G., Hyeon, T.: Nano Lett. 15, 4337-4342 (2015)

24. Mahmed, N., Heczko, O., Söderberg, O., Hannula, S.-P.: IOP Conf. Series: Mater. Sci. Eng. 18, 032020 (2011). doi:10.1088/1757-899X/18/3/032020 